\title{
SOLAR SAIL APPLICATION TO COMET NUCLEUS SAMPLE RETURN
}

\author{
Dr. Travis S. Taylor, Tryshanda T. Moton*, Don Robinson, R. Charles Anding \\ Teledyne Brown Engineering, Inc. \\ 300 Sparkman Drive \\ Cummings Research Park \\ Huntsville, AL 35805 \\ Dr. Gregory L. Matloff \\ Bangs/Matloff Aerospace Consulting Co. \\ 417 Greene Avenue \\ Brooklyn, NY 11216 \\ Dr. Gregory Garbe, Edward Montgomery \\ Solar Sail Propulsion Project \\ MSFC, TD 20 \\ Huntsville, AL 35812
}

\begin{abstract}
Many comets have perihelions at distances within 1.0 Astronomical Unit (AU) from the sun. These comets typically are inclined out of the ecliptic. We propose that a solar sail spacecraft could be used to increase the inclination of the orbit to match that of these 1.0 AU comets. The solar sail spacecraft would match the orbit velocity for a short period of time, which would be long enough for a container to be injected into the comet's nucleus. The container would be extended from a long durable tether so that the solar sail would not be required to enter into the potentially degrading environment of the comet's atmosphere. Once the container has been filled with sample material, the tether is retracted. The solar sail would then lower its inclination and fly back to Earth for the sample return. In this paper, we describe the selection of cometary targets, the mission design, and the solar sailcraft design suitable for sail-comet rendezvous as well as possible rendezvous scenarios.
\end{abstract}

\section{Selection of Cometary Targets Suitable for Solar Sail Rendezvous}

The concept of solar sail rendezvous missions has been studied for quite some time. During the 1970s, a design team at the Jet Propulsion Laboratory (JPL) discovered a trajectory that could allow a solar sail to rendezvous with Halley's Comet at its perihelion in the mid-1980s [1]. A rendezvous with Halley's Comet for that passage was looked at extensively by NASA scientists.
However a dedicated Comet Halley rendezvous mission was never flown by the United States.

Exploration of the mission-enabling properties of solar sailing to chase and rendezvous with comets continues as a small, although important focus of research interests. New comets approach the Sun from the outer regions of the solar system, beyond the orbit of Pluto. One of these comets shows up every few years. These comets are thought to

*AIAA Member, propulsion systems engineer

American Institute of Aeronautics and Astronautics 
typically have made only a few passes by the Sun and many are making their first approach to the Sun. These comets are thought to have remained essentially unchanged from the early formation of the solar system. As a result, comets and the matter that compose them are of very high interest for scientific research interests [2].

It is very likely that a solar sail spacecraft could be used to rendezvous with many comets that have perihelions close to Earth's orbit. Table 1 shows many short-period comets with perihelion less than or equal to $1.0 \mathrm{AU}$, which have been observed to have two or more apparitions [3]. The design effort for the Halley Rendezvous mission suggested a sail design that could operate at $0.25 \mathrm{AU}$ or closer from the Sun for at least 1 year without appreciable damage to the solar sail spacecraft. Technology development efforts on materials indicate that sailcraft capable of sustained operation inside 0.2 AU might be possible in the near future [2].

\begin{tabular}{|c|c|c|c|c|c|}
\hline Comet & $\frac{\text { Orbital Period }}{\text { (Years) }}$ & $\frac{\text { Perihelion }}{(\mathrm{AU})}$ & Eccentricity & $\frac{\text { Inclination }}{---}$ & $\frac{\text { Aphelion }}{\text { (AU) }}$ \\
\hline $\begin{array}{l}\text { 2P Encke } \\
\text { 107P Wilson- }\end{array}$ & 3.28 & 0.33 & 0.850 & 11.9 & 4.09 \\
\hline Harrington & 4.29 & 1.00 & 0.623 & 2.8 & 4.29 \\
\hline 26P Grigg-Skjellerup & 5.10 & 1.00 & 0.664 & 21.1 & 4.93 \\
\hline $\begin{array}{l}\text { 96P Machholz } \\
\text { 45P Honda-Mrkos- }\end{array}$ & 5.24 & 0.13 & 0.958 & 60.1 & 5.91 \\
\hline $\begin{array}{c}\text { Pajdusakeva } \\
\text { 73P Schwassman- }\end{array}$ & 5.30 & 0.54 & 0.822 & 4.2 & 5.54 \\
\hline Wachmann 3 & 5.35 & 0.94 & 0.694 & 11.4 & 5.18 \\
\hline 5D Brorsen & 5.46 & 0.59 & 0.810 & 29.4 & 5.61 \\
\hline 103P Hartley 2 & 6.26 & 0.95 & 0.719 & 9.3 & 5.84 \\
\hline $\begin{array}{l}\text { 3D Biela } \\
\text { 72P Benning- }\end{array}$ & 6.62 & 0.86 & 0.756 & 12.5 & 6.19 \\
\hline Fujikawa & 9.01 & 0.78 & 0.820 & 8.7 & 7.88 \\
\hline 8P Tuttle & 13.50 & 1.00 & 0.824 & 54.7 & 10.30 \\
\hline 27P Crommelin & 27.40 & 0.74 & 0.919 & 29.1 & 17.40 \\
\hline 55P Tempel-Tuttle & 32.90 & 0.98 & 0.904 & 162.7 & 19.60 \\
\hline 23P Brorsen-Metcalf & 70.50 & 0.48 & 0.972 & 19.3 & 33.70 \\
\hline 121P Pons-Brooks & 70.90 & 0.77 & 0.955 & 74.2 & 33.50 \\
\hline 1P Halley & 76.00 & 0.59 & 0.967 & 162.2 & 35.30 \\
\hline 109P Swift-Tuttle & 135.00 & 0.96 & 0.964 & 113.4 & 51.70 \\
\hline 35P Herschel-Rigollet & 155.00 & 0.75 & 0.974 & 64.2 & 56.90 \\
\hline
\end{tabular}

Table 1. Comets with Perihelion Close to Earth's Orbit

Table 1 shows that there are at least 18 comets that approach Earth's orbit. Nine of them have aphelions of 4.09-6.19 AU (these may have been influenced by Jupiter, although an analysis of this is beyond the scope of this paper). The 
table also shows that 8 have inclinations $0-20$ degrees, 4 have inclinations $21-$ 60.1 degrees, 3 have inclinations $61-100$ degrees, 3 have inclinations 101-180 degrees. The average inclination for the class is 47.3 degrees. The average perihelion for the class is 0.74 degrees. The average eccentricity for this comet class is 0.844 .

Comet velocity at various points along its orbit and sailcraft velocity at various orbital locations can be determined according to G. R. Fowles [4]. From Eq. (6.49) of this reference, we easily obtain an equation relating comet velocity at perihelion $\mathrm{V}_{\mathrm{o}}$ to circular-orbit velocity at perihelion $V_{c}$ and eccentricity e as

$$
V_{o}=V_{C}(e+1)^{1 / 2}
$$

Modifying Fowle's Eq. (6.51) to find perihelion velocity from perihelion solar separation $R_{0}$ and aphelion solar separation $R_{1}$ yields

$$
\begin{aligned}
V_{0} / V_{C}= & \left\{\left[2 R_{1} / R_{0}\right] /\right. \\
& {\left.\left[1+\left(R_{1} / R_{0}\right)\right]\right\}^{1 / 2} }
\end{aligned}
$$

Knowing the spacecraft velocity at perihelion and the perihelion distance, we can equate energy at perihelion to energy at any other solar distance $R_{\text {so }}$ (assuming no orbital energy change) to calculate orbital velocity at $R_{\text {so }}$, which is

$$
\begin{aligned}
V_{s o}= & {\left[V_{0}^{2}-2.65 \times 10^{20}\right.} \\
& \left.\left(R_{s o}-R_{o}\right) /\left(R_{s o} R_{o}\right)\right]^{1 / 2}
\end{aligned}
$$

\section{Comet and Sailcraft Orbits}

As comets approach the Sun, they have close to the energy needed to escape from the solar system. They typically have substantial inclinations from the ecliptic plane, which makes the comets difficult to reach in terms of energy requirements. Since some of these comets are discovered only a few months prior to their perihelion passage, it becomes essential to prepare a spacecraft for launch for this type of mission within a few months notice. However, high performance sailcrafts having characteristic accelerations $\left(a_{c}\right)$ greater than $5 \mathrm{~mm} / \mathrm{s}^{2}$, can reach some of these comets on short notice if the inclination of the comet's orbit is not too high and if the comet approaches from and acceptable direction relative to the position of the Earth in its orbit. If such a high performance sailcraft were available when the new comet approaches, a ready-made instrument package could be placed on the ship. In an ideal scenario, this would be done in a high orbit, such as L4 or L5. However, this might be accomplished by a tether deployment from a station that has taken a few weeks to gain the necessary escape energy to place it in this high orbit [2].

\section{Sample Mission Design for Solar Sail Rendezvous, Sample Return (SSRSR) to Comet 107P Wilson-Harrington}

\section{Rationale for Target Choice}

This object is chosen for a number of reasons. One, its perihelion is at $1 \mathrm{AU}$, which requires less spacecraft orbit adjustment. Two, it orbits the Sun every 4.29 years, allowing ample mission opportunities. Three, its inclination is 2.8 degrees, which also results in less maneuver requirements (less inclination changing). Also called Minor Planet 4015 , this comet's aphelion is $4.29 \mathrm{AU}$ and its orbital eccentricity is 0.623 [3]. Interestingly, Comet $107 \mathrm{P}$ was an alternate target for Deep-Space 1. As an Earth-crosser, it belongs to the class of 
objects, which sometimes strike the Earth. As a comet, its surface may contain biological progenitors (it is even speculated that primitive life may be found there). So a sample-return mission to this object may have large scientific and public support.

\section{Spacecraft Design}

Disc Sail: One possible architecture for the solar sail rendezvous-sample return (SSRSR) solar sailcraft is a disc sail. We assume a $50-\mathrm{kg}$ payload, a disc sail radius of $50 \mathrm{~m}$ (which implies a sail area of $7,850 \mathrm{~m}^{2}$ ). The sail-film areal mass density is $0.006 \mathrm{~kg} / \mathrm{m}^{2}$, and the structural mass factor is 0.3 . We assume a sail reflectivity $\left(\mathrm{REF}_{\text {sail }}\right)$ of 0.9 . The spacecraft areal mass density $\left(\sigma_{\mathrm{s} / \mathrm{c}}\right)$ is $0.012 \mathrm{~kg} / \mathrm{m}^{2}$.

First, we calculate sailcraft lightness factor, $\beta_{\mathrm{s} / \mathrm{c}}$ using Eq. (4.19) of Ref. 3

$$
\begin{gathered}
\beta_{s / c}=0.000787\left[\left(1+R E F_{s u l l}\right) /\right. \\
\left.\left(\sigma_{s / c}\right)\right]=0.12
\end{gathered}
$$

This means that the sail acceleration is $7.2 \times 10^{-5} \mathrm{~g}$ or $7.3 \times 10^{-4} \mathrm{~m} / \mathrm{sec}^{2}$ if the sail is oriented normal to the sun.

Square Sail: A second architecture we considered is the typical square sail. The sail is assumed to be $100 \mathrm{~m}$ on a side. The sail areal density is the same as that of the disc. The support booms for the square sail have a linear density of 50 $\mathrm{g} / \mathrm{m}$. The total lightness parameter for the square sail is about 0.12 and therefore has similar sail acceleration as for the disc sail.

Either of the above architectures will suffice for the SSRSR mission provided that the lightness parameter is on the order of 0.1 . It is the lightness parameter that drives the capability to perform the SSRSR mission. It should also be noted here that all materials suggested for the spacecraft architecture are currently available although state-of-the-art.

\section{The Launch Vehicle and Departure from Earth-Space}

A Delta-class expendable booster is the recommended launcher for the sailcraft. We suggest that the upper stage be powerful enough to supply an Earthescape (hyperbolic excess) velocity of about $3 \mathrm{~km} / \mathrm{s}$. This is the same hyperbolic excess required to insert an Earth-launched spacecraft into a Marsbound Hohmann-transfer ellipse [7]. A more capable upper stage supplying the $8 \mathrm{~km} / \mathrm{s}$ hyperbolic excess required to insert the spacecraft on a Jupiter-bound trajectory is, as will be discussed below, another option.

\section{Sail Maneuver 1: Inclination Change}

Our first sail maneuver is inclination change, using a curve-fit to Fig. 4.23 of McInnes [5]

$$
\begin{aligned}
\Lambda I / \Delta T & =\left(\beta_{s / c} / 0.05\right) \\
& \exp \left[-1.323 \ln \left(R_{s u}\right)-2.3\right]
\end{aligned}
$$

where $\Delta \mathrm{I} / \Delta \mathrm{T}$ is inclination change in degrees per week, and $\mathrm{R}_{\text {co }}$ is the constant cranking-orbit radius from the Sun during the inclination-change maneuver,

in Astronomical Units (AU). To alter inclination by 2.8 degrees to match the comet's inclination takes 12.7 weeks or 89 days. This maneuver will actually take a bit longer (about 10\%) since the $\mathrm{s} / \mathrm{c}$ is moving at a higher velocity than Earth's circular velocity after it departs Earth-space. 


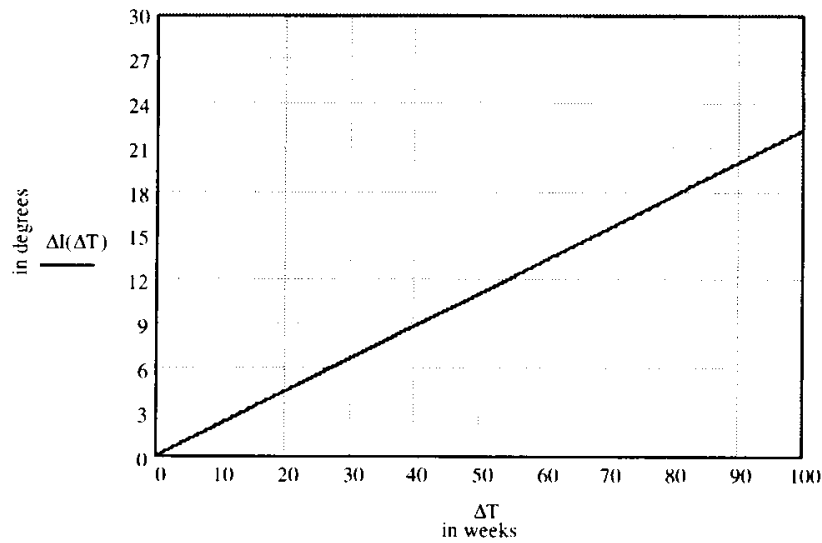

Figure 1. Inclination cranking versus time It is worth noting here other possible target missions and mission durations required. Figure 1 shows the inclination cranking achieved as a function of weeks. After 100 weeks an inclination of about 22 degrees could be reached. From Table 1 this shows that possibly 8 target comet orbits could be matched in about two years or less.

\section{Estimating Comet Perihelion Velocity}

From Equation 1 where $V_{o}$ is comet velocity at perihelion and $V_{c}$ is circular velocity at comet's perihelion, substituting Earth's circular velocity as the circular velocity at comet perihelion, and using $\mathrm{e}=0.623$, we find that the comet's perihelion velocity relative to the Sun is $38 \mathrm{~km} / \mathrm{s}$, or about $8 \mathrm{~km} / \mathrm{s}$ faster than the Earth at perihelion and 5 $\mathrm{km} / \mathrm{s}$ faster than the spacecraft in its post-earth-escape solar orbit.

\section{Comet Rendezvous Alternative 1: A Holographic Sail}

This rendezvous alternative assumes a tangential component of sailcraft acceleration after the inclinationcranking maneuver. Elementary kinematics reveals that the spacecraft can match the comet's perihelion velocity in 0.5 years if it has a tangential acceleration of $3.17 \times 10^{-4} \mathrm{~m} / \mathrm{s}^{2}$, less than $1 / 2$ of its solar-radiation-pressure acceleration at $1 \mathrm{AU}$ if it is oriented normal to the Sun.

From Forward's "grey solar sail " paper [6], tangential acceleration can be written as

$$
\begin{aligned}
A C C_{t}= & {\left[\left(R E F_{\text {sail }}+\alpha+R_{b}-R_{s}\right) \sin \theta\right.} \\
& \cos \theta S) /\left(c \sigma_{s / c}\right),
\end{aligned}
$$

where $\alpha=$ sail absorptance, $R_{b}$ is sail back (mirror-like) reflectance, $R_{\mathrm{s}}$ is sail specular reflectance, $\theta$ is the sail-Sun aspect angle ( 0 degrees for sail normal to Sun), $S$ is the solar flux in watts per square meter, and $\mathrm{c}$ is the speed of light. For a specular sail, the tangential acceleration component will be very low.

But if the sail is back-reflective, we can apply Forward's Eq. (50) from ref. 6 to obtain

$$
A C C_{t}=A C C_{s / c} \sin \theta \cos \theta,
$$

where $\mathrm{ACC}_{\mathrm{s} / \mathrm{c}}$ is the sailcraft acceleration if oriented normal to the Sun. In this case, an angle of 45 degrees between normal to the sail and the Sun results in a tangential acceleration of $3.25 \times 10^{4}$ $\mathrm{m} / \mathrm{s}^{2}$, which is more than enough.

Forward suggests that we might obtain a mirror-like sail coating by embossing the sail reflective layer with images of corner-cube reflectors [8]. This might be done holographically [9].

\section{Comet Rendezvous Alternative 2: High-} Energy Upper Stage

This alternative replaces the tangential acceleration by sail near perihelion with an upper stage capable of leaving Earthspace at a hyperbolic-excess velocity of $8 \mathrm{~km} / \mathrm{s}$ [4]. The sail is only used during 
the pre-rendezvous phase of the mission for inclination cranking. Interestingly, this approach has been used before-for the launches of Pioneer 10 and 11 and Voyager 1 and 2 in the 1970's. If this option is selected, more time must be devoted for the inclination-cranking maneuver.

Comet Rendezvous Alternative 3: Optimized Sailing Application

This approach applies results of an optimized-sail trajectory analysis reported on pp 139-140 of McInnes [1]. In the results of this computer simulation, a sail with $\beta_{\mathrm{s} / \mathrm{c}}=0.05$ departs a Sun-centered circular orbit at $1 \mathrm{AU}$ to a high-eccentricity solar elliptical orbit. The spacecraft requires 3 orbits of the Sun to increase its semi-major axis to 3 $\mathrm{AU}$, which we require for comet rendezvous. Since acceleration scales with increasing $\beta_{\mathrm{s} / \mathrm{c}}$, we can do it with our sailcraft configuration in 1.5 orbits or about 5 years.

\section{Sample Collection}

Previous missions describe comet sample collection by placing solar sail probes into orbits where they pass as close to the surface of the comet nucleus as the thermal capacity of the probe will allow. The system proposed here utilizes a similar concept of releasing a sample capturing device such as a capsule roughly the size of a 12-ounce soda can attached to the sailcraft by a tether (possibly Spectra $1000^{\mathrm{TM}}$ ). Once the sailcraft is within the orbit of the comet, a tether system will be spring launched toward the comet to pick up a sample of matter from the comets nucleus. The sailcraft will be capable of releasing the tethered capsule and then altering its trajectory to avoid incineration or any other impact from the comet's matter that may potentially damage the solar sail. The capsule will then be closed and retracted and a sample of the comet's nucleus matter is returned to scientists for examination back on Earth.

Considering the reference mission design discussed thus far, the sample return spacecraft will match velocities with the 107P/Wilson-Harrington comet. Matching velocities reduces the risk of particle impacts since it is likely to only incur very minor damage and slight course alterations from low velocity gas and particle collisions. If the relative velocity of spacecraft to dust is high, problems could occur similar to the European Space Agency (ESA) Giotto mission which intercepted Halley's comet in March of 1986 [10]. Giotto received significant course alteration and damage. Fourteen seconds before closest approach Giotto was hit by a 'large' dust particle which caused the spacecraft angular momentum vector to shift 0.9 degrees. The primary concern for SSRSR is likely to be the possibility of gas jets erupting from the comet with course altering force. However, some of the comets we are considering are somewhat inactive, including $107 \mathrm{P} /$ Wilson-Harrington according to the work and assessment by Marsden [11], "the observations suggest that the object is a largely inactive comet that undergoes occasional outburst."

The spacecraft will utilize cameras and built in digital signal processing techniques to analyze the surface for an optimal landing location. The mission may last weeks, which will allow the Comet Mission Earth Team to make the final decision of the landing site. If any of the potential landing sites show gas 
jet activity, landing will be attempted in some other area. The soda can sized lander will separate with the gentle release of a spring-loaded mechanism and will begin to move away from the main body of the sailcraft very slowly. A tether reel on the sailcraft will maintain approximately zero tension as the tether reels out at a speed matching the lander's speed. The tether will also have a fiber optic cable wound around it that will enable direct optical communication between the sailcraft and the lander's main computer. The main computer on the sailcraft will use data from the lander's proximity detector to control very small cold gas thrusters for a proper comet relative velocity touchdown at a selected comet landing site. The current assumption is that the comet velocity matching will not leave a significant comet rotational component relative to the sailcraft and subsequently the lander.

The soda can lander will adhere to the comet by implementing synthetic Gecko skin coated landing pads. Geckos are small reptiles whose feet have hundreds of thousands of hair-like "setae" with hundreds of submicroscopic pads ("spatulae") at each seta tip, which appear to cling by van der Waals forces to almost any surface even while under conditions of vacuum and particulate contamination. Experiments and analyses have been conducted that suggest the skin can be synthesized and may deliver adhesion forces of as much as $10 \mathrm{~N}$ per $100 \mathrm{~mm}^{2}$ [12]. The lander will simply bump into the comet nucleus and the pads will adhere to the surface.

The comet nucleus is sampled via a "sampling tool" which is a counterrotating drill-like system. The sampling tool will rotate slowly and be pulled deeper into the comet nucleus as it rotates. The counter-rotation will produce almost zero net torque to help minimize the probability that the comet nucleus section being drilled will break away in an uncontrolled manner. The comet nucleus material is collected in a comet nucleus material bag. When the desired size of the comet material sample has been obtained the drilling portion of the sampling tool will be ejected as a volume exchange and the material bag is sealed. The sampling tool ejection mechanism will also provide an external seal where it was previously housed and provides additional protection for the material bag.

In order to obtain separation of the lander from the comet the lander will release the Gecko skin landing pads and leave them on the comet in much the same way the Lunar Excursion Modules left the landing gear behind. The initial separation from the comet is powered by stored energy in a spring mechanism. The lander computer will send a signal to sailcraft indicating a successful separation and the sailcraft will then reel in the tether. The maximum tension expected on the tether will be due to the force necessary to accelerate the approximately $10 \mathrm{~kg}$ lander and full sample material bag to the sailcraft velocity. Development of a sample storage system for the flight back to Earth must be considered if ice particles are to be maintained. A detailed analysis of the environmental requirements necessary to maintain the sample should be conducted, but is beyond the scope of this paper. 


\section{Earth Return}

The sailcraft will fly the sample back to Earth by undoing the orbit and inclination changes discussed previously. Once the sailcraft approaches Earthspace it is possible that it can survive an aerobraking encounter with the Earth whereas its sail is used as the aerobrake ballute. Therefore only inclination-cranking and minor maneuvers may be required on the Earthbound leg. The aerobraking maneuver could leave the sail in an elliptical orbit around Earth with its periapsis at a low enough orbit that the Shuttle could capture the sample and return it to Earth. It is also possible that if the periapsis is too high for Shuttle capture that the soda can lander could be launched toward Earth using a stored energy spring or cold gas thruster. The tether would be reeled out in order for the Shuttle to capture it. This should be investigated further.

\section{Conclusion}

We have shown here a concept mission architecture for a comet nucleus sample return using a solar sail rendezvous. A small tethered soda can spacecraft could then be used to capture a sample of the comet's nucleus and return it to Earth. The sailcraft would return the sample to Earthspace whereas the sail is used for an aerobrake. The sample could then be returned to Earth via the Space Shuttle. The analysis presented here suggests that a solar sail spacecraft is ideal for the comet nucleus sample return. More detailed studies for the mission concept should be conducted.

\section{References}

1. C. R. McInnes, Solar Sailing, Springer-Praxis, Chichester, UK (1999), pp.4-11; pp 139-140.

2. Wright, J. L., Space Sailing, Gordon and Breach Science Publishers, PA, (1992), pp.42-45.

3. R. P. Binzer, M. S. Hanner, and D. I. Steel, "Solar System Small Bodies,"A. N. Cox ed., Allen's Astrophysical Quantities, $4^{\text {th }}$. ed., Springer-Verlag, NY (2000)

4. G. R. Fowles, Analytical Mechanics, Holt, Rinehart, and Winston, NY (1962).

5. N. C. Wickramsinghe, F. Hoyle, S. Al-Mufti, and D. h. Wallis, "Infrared Signatures of Prebiology—or Biology," in Astronomical and Biochemical Origins and the Search for Life in the Universe, ed. C. B. Cosmovici, S. Bowyer, and D. Werthimer, Editrice Compositori, Bologna, Italy (1997), pp 61-76.

6. G. L. Matloff, Deep-Space Probes, Springer-Praxis, Chichester, UK (2000).

7. R. R. Bate, D. D. Mueller, and J. E. White, Fundamentals of Astrodynamics, Dover, NY (1971), pp. 362-365.

8. R. L. Forward, "Grey Solar Sails," J. Astronaut. Sci., 38, 161-185 (1990); also published as AIAA 89-2343.

9. G. L. Matloff, G. Vulpetti, C. Bangs, and R. Haggerty, "The Interstellar Probe (ISP) : PrePerihelion Trajectories and Application of Holography, "NASA / CR- 2002-211730, NASA/MSFC, Huntsville Alabama (2002). 
10. D.R. Williams, http://nssdc.gsfc.nasa.gov/planeta ry/giotto.html, 2003.

11. B. G. Marsden, http://cometography.com/pcomet s/107p.html, 2003.

12. Autumn, K., et al. (2000). Adhesive force of a single gecko foot-hair." Nature, 405, pp. 681685 . 